\title{
RELATION OF WHITEHEAD AND RUSSELL'S THEORY OF DEDUCTION TO THE BOOLEAN LOGIC OF PROPOSITIONS*
}

\author{
BY B. A. BERNSTEIN
}

1. Introduction. Whitehead and Russell's theory of deduction for "elementary" propositions, which underlies their Principia Mathematica, is "the calculus of propositions"; it is "the theory of how one proposition can be inferred from another"; the primitives of the theory "are sufficient for all common forms of inference." The theory thus seems to be designed to yield the same facts of logic as are given by the classic Boolean logic of propositions. But our authors tell us that "the theory of classes and the theory of propositions . . . are not coordinate," that "the theory of propositions necessarily precedes the theory of classes." The theory of deduction then seems to be essentially different from the Boolean logic of propositions. The latter logic is coordinate with the logic of classes; it in no way precedes the logic of classes. Just what is the mathematical relation of the theory of deduction to the Boolean logic of propositions? The object of my paper is to answer this question.

2. Boolean Form of the Theory of Deduction. A mathematical comparison between the theory of deduction and the Boolean logic of propositions requires that the two logics be expressed in a common mathematical language. This common language I have provided in a previous paper $\dagger$, when I rewrote the theory of deduction in the language of Boolean algebra. I begin my discussion by presenting the essentials of the two logics in Boolean form.

In Boolean form, the theory of deduction is a system $\left(K,{ }^{\prime},+\right)$, consisting of an undefined class $K$, an undefined unary operation "'", and an undefined binary operation "+", satisfying the following eight postulates:

* Presented to the Society, June 20,1929, as part of a paper entitled On Whitehead and Russell's theory of deduction.

$\dagger$ Whitehead and Russell's theory of deduction as a mathematical science, this Bulletin, vol. 37 (1931), pp. 480-488. 
1.1. There exists a $K$-element 1 such that from $a=1$ and $a^{\prime}+b=1$ follows $b=1$.

1.2. $(a+a)^{\prime}+a=1$.

1.3. $b^{\prime}+(a+b)=1$.

1.4. $(a+b)^{\prime}+(b+a)=1$.

1.5. $[a+(b+c)]^{\prime}+[b+(a+c)]=1$.

1.6. $\left(b^{\prime}+c\right)^{\prime}+\left[(a+b)^{\prime}+(a+c)\right]=1$.

1.7. If $a$ is a $K$-element, $a^{\prime}$ is a $K$-element.

1.71. If $a$ and $b$ are $K$-elements, $a+b$ is a $K$-element.

The important defined ideas in the theory are $a \supset b, a \cdot b$ (or $a b), a \equiv b$. The definitions are

DEF. 1.01. $(a \supset b)=a^{\prime}+b$,

DEF. 3.01. $a b=\left(a^{\prime}+b^{\prime}\right)^{\prime}$,

DEF. 4.01. $(a \equiv b)=(a \supset b)(b \supset a)=\left(a^{\prime}+b\right)\left(b^{\prime}+a\right)$.

3. The Boolean Logic of Propositions. The Boolean logic of propositions may be characterized as a system $(K,+)$, consisting of an undefined class $K$ and an undefined binary operation "+", satisfying the following three postulates*:

A. There is a $K$-element 0 such that $0+a=a$ for every $K$-element $a$.

B. There is a $K$-element 1 such that $1+a=1$ for every $K$-element $a$.

C. $K$ consists of two elements.

The important defined ideas of this system are $a^{\prime}, a+b$ (or $a b), a<b$. The definitions are:

DEF. $1.0^{\prime}=1,1^{\prime}=0$,

DEF. 2. $a b=\left(a^{\prime}+b^{\prime}\right)^{\prime}$,

DEF. 3. $(a<b)=\left(a^{\prime}+b=1\right)$.

* See my Sets of postulates for the logic of propositions, Transactions of this Society, vol. 28 (1926), pp. 472-478.

$\dagger$ Note that $a<b$ is not the same as $a \supset b$ of $\$ 2$. As a concrete science, $(K,+)$ is an algebra of "truth-values," truth 1 and falsity 0 . Let $p, q$ be two propositions and $a, b$ their respective truth-values. Then $a^{\prime}, a+b, a b$ are the respective truth-valves of the propositions "not $-p$," " $p$ or $q$," " $p$ and $q$ "; the relation $a<b$ signifies " $p$ implies $q$," and $a=b$ signifies " $p$ is equivalent to $q . "$ 
The operations $a+b$ and $a b$ and the relation $a<b$, as determined by postulates A-C and definitions 2,3 , are given by the following tables:*

\begin{tabular}{c|cc}
+ & 0 & 1 \\
\hline 0 & 0 & 1 \\
1 & 1 & 1
\end{tabular}

(ii) \begin{tabular}{c|cc}
$\times$ & 0 & 1 \\
1 & 0 & 0 \\
0 & 1
\end{tabular} (iii) \begin{tabular}{c|cc}
$<$ & 0 & 1 \\
\hline & + & + \\
1 & +
\end{tabular}

Each of these tables shows that the Boolean logic of propositions is a two-element logic of classes.

4. The Theory of Deduction Derivable from the Boolean Logic of Propositions. Now that we have before us the theory of deduction and the Boolean logic of propositions expressed in a common language, I can proceed to answer the question, raised in $\S 1$, concerning the relation between the two theories. I observe, first, that the theory of deduction is derivable from the Boolean logic of propositions.

This fact can easily be verified by deriving propositions 1.1 1.71 of $\S 2$ from propositions $\mathrm{A}-\mathrm{C}$ of $\S 3$, or by using table (i) of $\S 3$. As illustration of the use of table (i), I give the proofs of 1.1 and 1.6, perhaps the least simple of the proofs.

Proof of 1.1. The element 1 in postulate $B$ is the element 1 required in 1.1. For suppose $a=1$ and $a^{\prime}+b=1$, that is, suppose $1^{\prime}+b=1$. Then $b=1^{\prime}+b=1$, by table (i), def. 1, hyp.

Proof of 1.6. (1) $a^{\prime}+a=1$, by def. 1 and table (i).

Further, for $a=0$, the left member of 1.6 is

(2) $\left(b^{\prime}+c\right)^{\prime}+\left[(0+b)^{\prime}+(0+c)\right]=\left(b^{\prime}+c\right)^{\prime}+\left(b^{\prime}+c\right)=1$,

by table (i) and (1).

For $a=1$, the left member of 1.6 is

$$
\begin{aligned}
\left(b^{\prime}+c\right)^{\prime}+\left[(1+b)^{\prime}+(1+c)\right] & =\left(b^{\prime}+c\right)^{\prime}+\left(1^{\prime}+1\right) \\
=\left(b^{\prime}+c\right)+1 & =1
\end{aligned}
$$

by table (i), (1), table (i).

Hence the theorem follows, by (2) and (3).

* In reading the tables, take $a$ from the extreme left column and $b$ from the uppermost row. In table (iii) a " + " sign denotes that $a<b$ holds, a "-" sign that $a<b$ does not hold. 
5. The Boolean Logic of Propositions Not Derivable from the Theory of Deduction. I observe, next, that the Boolean logic of propositions is not derivable from the theory of deduction.

For, the following system $\left(K,{ }^{\prime},+\right)$ is an independence-system for proposition $\mathrm{C}$ with respect to 1.1-1.71:

$K$, a class consisting of the single element $e ; e^{\prime}=e ; e+e=e$.

Of course, all propositions in the Boolean logic of propositions that are dependent on $\mathrm{C}$ are propositions not derivable from the theory of deduction. Among such propositions, for example, are the following:

D. For every $K$-element $a$, either $a=1$ or else $a=0$.

E. $a^{\prime} \neq a$.

F. If $a<b$ or $a<c$ then $a<b+c$, and conversely.

That D-F are propositions in the Boolean logic of propositions can easily be verified with the help of table (i).

That $\mathrm{D}$ is not derivable from $1.1-1.71$ is seen from the following independence-system $\left(K,{ }^{\prime},+\right)$ :

$K$, the infinite set of plane regions contained in a plane region $T$, including $T$ and including the null region $N ; a^{\prime}$, the region in $T$ outside $a ; a+b$, the smallest region containing $a$ and $b$.

An independence-system for $E$ with respect to $1.1-1.71$ is the independence-system for $\mathrm{C}$ above.

An independence-system for $\mathrm{F}$ is the system used in proving the independence of $D{ }^{*}$

6. Inadequacy of the Theory of Deduction as "the Calculus of Propositions." The fact that propositions D-F are unobtainable from 1.1-1.71 shows that the theory of deduction is inadequate as "the calculus of propositions." The theory of deduction is "the theory of how one proposition can be inferred from another"; its primitives "are sufficient for all common forms of inference"; it is concerned with "the principles by which conclusions are inferred from premisses"; it is designed to yield "the rules" from which "the usual calculus of formal logic starts." The theory, as "the calculus of propositions," bears the same name as Schröder's Der Aussagenkalkül. But the theory of de-

* Compare my paper On proposition *4.78 of Principia Mathematica, this Bulletin, vol. 38 (1932), pp. 388-391. 
duction cannot yield such propositions as D-F above, propositions which are fundamental in the classic theory $\dagger$ and which the theory of deduction aims to obtain. $f$ Clearly, the theory of deduction is inadequate as "the calculus of propositions."

7. Conclusion. I have answered completely the question of the relation of the theory of deduction of the Principia to the Boolean logic of propositions. The theory of deduction is derivable from the Boolean logic of propositions, but not conversely. The latter fact makes the theory inadequate as "the calculus of propositions."

\section{The University of California}

$\dagger$ Proposition D is of particular importance in the classic theory. For D is essentially the same as proposition $\mathrm{C}$ above, and $\mathrm{C}$, as I have shown elsewhere, can be made to serve as a one-postulate set for the Boolean logic of propositions. (See my Sets of postulates for the logic of propositions, cited above.)

$\ddagger$ The authors of the Principia no doubt regard D-F as equivalent to their

* 2.11. $\vdash \cdot p \bigvee \sim p$,

* 5.19. $\sim \sim(p \equiv \sim p)$,

* 4.78. $\vdash \cdot[(p \supset q) \bigvee(p \supset r)] \equiv[p \supset(q \bigvee r)]$

For they would interpret these propositions respectively as:

$D_{0}$. Every proposition is true or else false,

$\mathrm{E}_{0}$. A proposition is never equivalent to its contradictory,

$\mathrm{F}_{0}$. If $p$ implies $q$, or $p$ implies $r$, then $p$ implies " $q$ or $r$ "; and conversely. Interpretations $\mathrm{D}_{0}-\mathrm{F}_{0}$ are, however, all inadmissible. They are based on reading the fundamental symbols $\sim p, p \bigvee q, p \supset q, p \cdot q, p \equiv q$ respectively as " $p$ is false," " $p$ is true or $q$ is true," " $p$ implies $q$ ", " $p$ is true and $q$ is true," " $p$ is equivalent to $q$ "-readings which all violate the distinction between " $p$ " and " $\vdash \cdot p$ " made by the authors earlier in their theory. The correct readings of the fundamental symbols should be, respectively, "not $-p$, , " $p$ or $q$," "not $-p$ or $q$," " $p$ and $q$," " not $-p$ or $q$ ' and 'not $-q$ or $p$ ". (Compare the remarks on "ト" and " $\supset$ " in my review of Volume I of the Principia, this Bulletin, vol. 32 , pp. $711-713$. With regard to $* 4.78$, compare also my paper on $* 4.78$, cited above.) 